\title{
Displacement of deciduous tooth into hypopharynx due to endotracheal intubation
}

\author{
Sang-Hoon Kang ${ }^{1}$, Jung Hyun Chang $^{2}$ \\ Departments of ${ }^{1}$ Oral and Maxillofacial Surgery, ${ }^{2}$ Otorhinolaryngology, National Health Insurance Service Ilsan Hospital, Goyang, Korea
}

\begin{abstract}
Intubation may lead to several dental complications. Furthermore, a tooth damaged during intubation may be subsequently dislocated. In the present case, the upper primary incisor was avulsed during intubation and, unbeknownst to the anesthesiologist, displaced to the larynx. We report here on the findings and indicate appropriate treatment. Intubation for general anesthesia in children can result in tooth damage and/or dislocation of primary teeth with subsequent root resorption. Prevention is key, and thus it is critical to evaluate the patient's dental status before and after intubation. Furthermore, anesthesiologists and dentists should pay close attention to this risk to prevent any avulsed, dislocated, or otherwise displaced teeth from remaining undetected and subsequently causing serious complications.
\end{abstract}

Key Words: Anesthesia; General; Intubation; Tooth injuries.

Intubation may lead to several dental complications [1-3]; for example, using a laryngoscope for intubation may damage teeth. Furthermore, intubation may cause teeth dislocated due to trauma to be displaced into the bronchus [4]. Moreover, a tooth damaged as a result of intubation may be dislocated [5]. In the present case, the upper primary incisor was avulsed during intubation and subsequently displaced to the larynx without the anesthesiologist's knowledge. We report here on the findings and appropriate treatment.

\section{CASE REPORT}

A six-year-old girl was transferred to our hospital with an impacted supernumerary tooth in the upper anterior maxillary region. Other than being born via caesarean section at 38 weeks, her medical and dental history were unremarkable. Panoramic radiographic imaging showed a supernumerary tooth impacted in the upper anterior region (Fig. 1). We decided to surgically extract this tooth via the palatal side. Although it was possible to perform the extraction under local anesthesia, as the depth of the impaction was not severe, general anesthesia was recommended because the patient had previously been uncooperative during treatment. However, the patient's mother requested the procedure be performed under local anesthesia. Prior to treating the patient, we attempted to

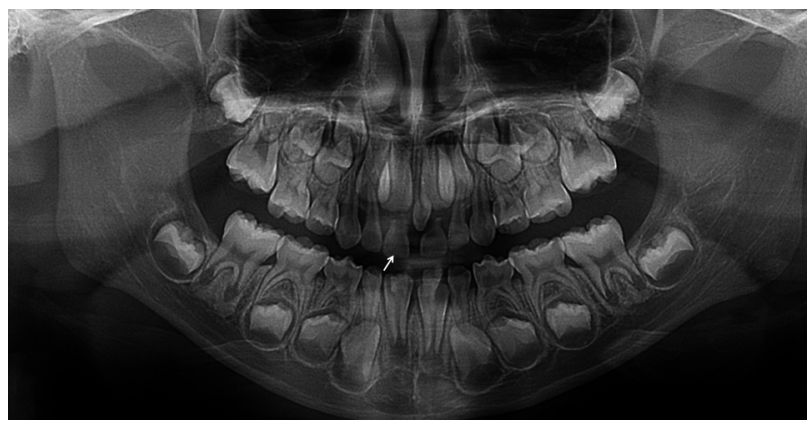

Fig. 1. Preoperative panoramic radiograph. Deciduous incisor (arrow) displaced into the laryngopharyngeal area after endotracheal intubation.
Copyright(c) 2016 Journal of Dental Anesthesia and Pain Medicine
Received: 2016. February. 17. • Revised: 2016. March. 11. • Accepted: 2016. March. 16. Corresponding Author: Jung Hyun Chang, Department of Otorhinolaryngology, National Health Insurance Service Ilsan Hospital, 100 Ilsan-ro, Ilsan-donggu, Goyang, Gyeonggi-do, 410-719, Korea Tel: +82-31-900-0346 eFax: +82-303-3448-7138 E-mail: manbang5@naver.com 
perform infiltration anesthesia after applying anesthetic topical agents on the upper anterior maxillary region, but the patient closed her mouth and refused to cooperate with the treatment. As a result, we could not treat the patient that day. The patient and her mother wanted to attempt the treatment again under local anesthesia, so it was postponed. During the subsequent appointment, we again attempted local anesthesia, but again, the patient did not cooperate. Thus, it was necessary to perform the supernumerary extraction under general anesthesia. The patient was $119 \mathrm{~cm}$ tall and weighed $21 \mathrm{~kg}$. Prior to intubation, the patient was intravenously administered $0.3 \mathrm{mg}$ remifentanil, $10 \mathrm{mg}$ rocuronium, and $50 \mathrm{mg}$ propofol. During intubation, we used a laryngoscope to improve the view of the larynx region. A 5-mm-diameter wire guide cuffed tube was intubated through the nasal cavity, and the length at the nostril was fixed to $20 \mathrm{~cm}$ of the tube. Then, we attached the tube to the patient's head, so it would not move during the operation. Whilst disinfecting the oral cavity after draping, we observed that the upper right primary incisor was missing. We asked the anesthesiologist whether the incisor was stuck to either the laryngoscope or Magill forceps, but were told that it was not. We also checked inside the oral cavity but could not see the missing primary incisor. To confirm whether the tooth was displaced to either the larynx region or bronchus, we took a plain radiograph of the

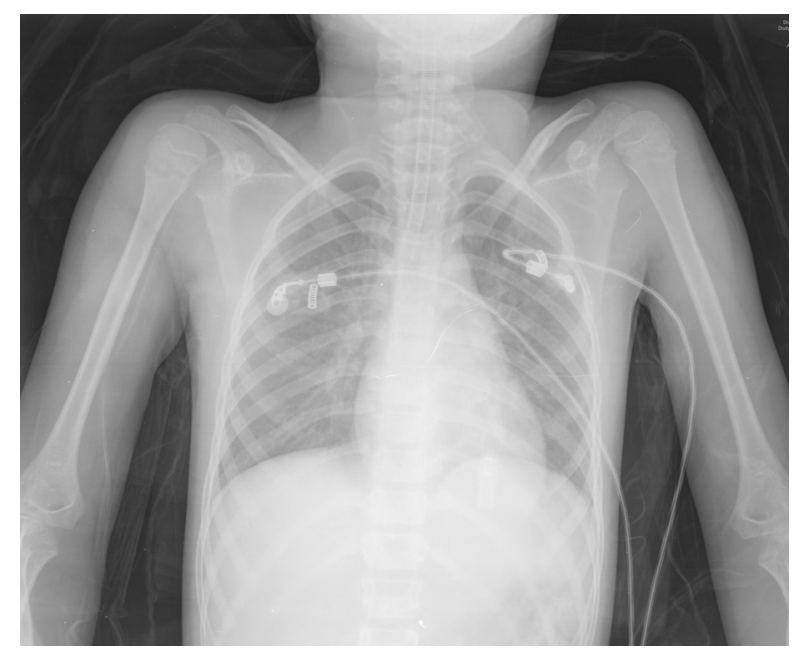

Fig. 2. Plain AP chest radiograph. lateral neck and anteroposterior (AP) chest. The lost tooth was not observed in the AP chest radiograph (Fig. 2). However, the lateral radiograph showed that it lay near the intubation tube in the pharynx (Fig. 3). We fixed the patient's head to prevent further displacement of the tooth and consulted the ear, nose, and throat (ENT) department for removal of the tooth. The ENT doctor confirmed the position of the displaced primary incisor using an endoscope. Then, he pulled the tongue down and fixed it using a MacGyver retractor to secure a clear view of the larynx region and increase accessibility (Fig. 4A). Next, we used suction and tonsil forceps to remove the displaced tooth from its location, near the intubation tube. After recovering the displaced tooth, we pulled back the MacGyver retractor and continued with the supernumerary tooth extraction procedure. The patient did not show any abnormal symptoms or complications after the procedure.

\section{DISCUSSION}

In the present case, a primary incisor was dislocated and subsequently displaced during anesthesia, but this was discovered prior to the surgery, allowing us to appropriately locate the displaced tooth via imaging and

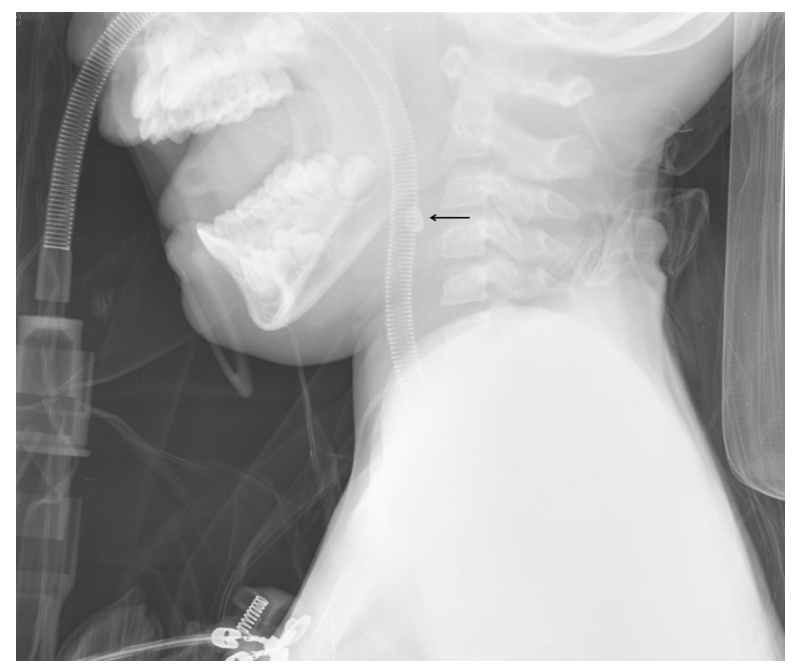

Fig. 3. Plain lateral neck radiograph indicated the lost deciduous incisor (arrow) lay near the intubated tube in the pharynx. 

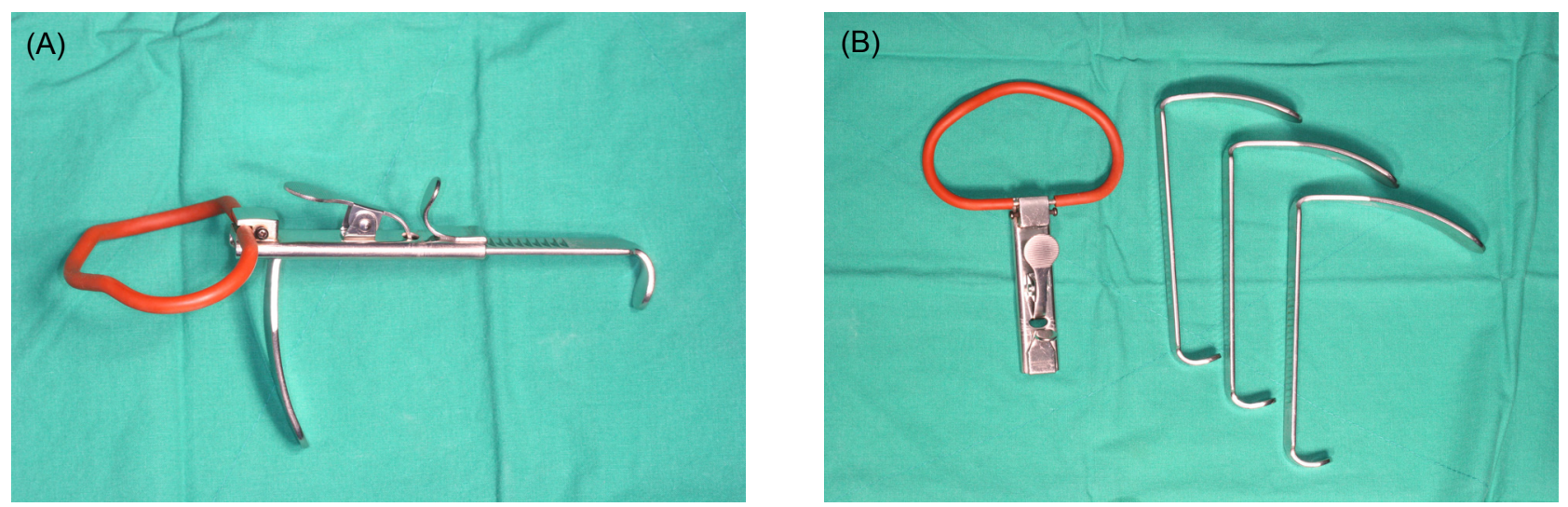

Fig. 4. (A) MacGyver retractor was used to pull the tongue down to secure a clear view of the larynx region and increase accessibility. (B) The MacGyver retractor can be used with tongue blades of various sizes depending on the size of the patient's tongue, and the degree of retraction can also be manipulated and maintained.

remove it after securing a view of the pharyngeal region. Supernumerary teeth are found during regular dental check-ups or through panoramic radiography imaging when patients visit the clinic for treatment of other teeth. There are cases in which a residual primary tooth is found during an extraction of a supernumerary tooth. There are also cases in which a permanent central incisor erupts and a supernumerary tooth is found. In these cases, there is a possibility of the maxillary tooth being dislocated upon trauma. For primary teeth, the root may be absorbed, whereas for permanent teeth, the root may still be forming; thus, intubation must be conducted with great care to avoid dislocation. Trauma to the permanent teeth in the anterior region is a known complication during general anesthesia $[2,3]$. Thus, the teeth in the upper anterior region must be assessed before and after intubation for general anesthesia $[2,6]$. The possibility of damage to a tooth, such as a fracture, and/or dislocation must be considered when using general anesthesia in children $[7,8]$.

If it is discovered that a tooth has been dislocated after intubation, it must be located and removed. For oral surgeries, intubation may be performed through the nasal cavity, and Magill forceps can manipulate the position of the tube. In such cases, the dislocated tooth may stick to the forceps and be removed with them. In addition, the oral cavity should also be viewed to try and locate the dislocated tooth. If the tooth still cannot be located, a radiograph should be taken in the operating room. Lateral and AP radiographs of the neck will indicate whether the tooth has been displaced to the larynx region. A plain AP chest radiograph is will determine whether the tooth has been displaced to the bronchus. In the present case, the dislocated tooth was not found in the $\mathrm{AP}$ chest radiograph and an AP neck radiograph was not taken, although it would have been helpful for determining the horizontal position of the tooth in the neck. If such dislocation occurs again, AP and lateral radiographs of the neck will be taken along with an AP chest radiograph. Once the position of the displaced tooth is determined, the possible methods of removal will need to be reviewed. It may be necessary to confirm the position of the tooth via a fiberoptic endoscope. In this case, soft tissues in the neck, including the tongue, should not be retracted, to avoid further displacement of the tooth.

If the dislocated tooth is found in the nasopharynx region, the tooth may be removed by retracting the uvula to the superior. If it has been displaced to the laryngopharynx region, as in this case, retracting the tongue to the inferior position is helpful in securing a view of the area. When using a laryngoscope, the tongue should still be retracted; however, the size and angle of the oral space may not be appropriate for removing the tooth. In the 
present case, a MacGyver retractor was used to secure a clear view and widen the oral and laryngopharynx space, which allowed easy removal of the tooth. The MacGyver retractor can be used with tongue blades of various sizes, depending on the size of the patient's tongue, and the degree of retraction can also be manipulated and maintained (Fig. 4B). The frame is supported by the premolar or hard palate, and the lower part retracts the tongue. The direction of the retractor can be adjusted to hold the tongue to the right or left, according to the position of the tooth, allowing a wider view of the area. The frame should be supported properly because if it is supported by the upper anterior region, the lateral force can damage the tooth. Primary teeth are small, so an appropriate device, such as strong suction, is required to hold the tooth.

If the tooth is found in the bronchus, a bronchial endoscope is used to remove it [9]. However, bronchial endoscopy in children may result in a series of complications. Hence, before proceeding, an adequate explanation must be given to the caregiver, other medical departments, such as pediatrics and pulmonary medicine should be consulted, appropriate surgical preparations should be made, and proper response measures should be planned in case of complications. There are methods recommended during general anesthesia to prevent dental injury $[10,11]$. A video laryngoscope may be used in the intubation procedure instead of a traditional laryngoscope $[11,12,13]$ and a dental protector may also be used $[10,14]$.

Extraction of supernumerary tooth in the upper anterior region in children is usually performed under local anesthesia or conscious sedation, neither of which require intubation. However, regardless of the method of anesthesia, the possibility of tooth dislocation remains when tooth mobility is severe. Intubation for general anesthesia during supernumerary extraction in children can result in tooth damage and dislocation of primary teeth with root resorption or permanent central incisors, which have incomplete root formation. Prevention is key; thus it is critical to evaluate the patient's dental status before and after intubation.
Furthermore, anesthesiologists and dentists must pay close attention to this risk to prevent any avulsed, dislocated, or otherwise displaced teeth from remaining undetected and subsequently causing serious complications.

\section{REFERENCES}

1. Chadwick RG, Lindsay SM. Dental injuries during general anaesthesia. Br Dent J 1996; 180: 255-8.

2. Warner ME, Benenfeld SM, Warner MA, Schroeder DR, Maxson PM. Perianesthetic dental injuries: frequency, outcomes, and risk factors. Anesthesiology 1999; 90: 1302-5.

3. Adolphs N, Kessler B, von Heymann C, Achterberg E, Spies C, Menneking $\mathrm{H}$, et al. Dentoalveolar injury related to general anaesthesia: a 14 years review and a statement from the surgical point of view based on a retrospective analysis of the documentation of a university hospital. Dent Traumatol 2011; 27: 10-4.

4. Casap N, Alterman M, Lieberman S, Zeltser R. Enigma of missing teeth in maxillofacial trauma. J Oral Maxillofac Surg 2011; 69: 1421-9.

5. Tammara A, Reed RM, Verceles AC. A missing tooth after intubation. BMJ Case Rep 2014; 2014.

6. Chidyllo SA, Zukaitis JA. Dental examinations prior to elective surgery under anesthesia. N Y State Dent J 1990; 56: 69-70.

7. Boye TA, Orr RL, Majdneya J. Spontaneous exhalation of an inhaled tooth in a child: a case report. Int J Oral Maxillofac Surg 2009; 38: 1333-5.

8. Lindemeyer RG, Rashewsky SE, Louie PJ, Schleelein L. Anesthetic and dental management of a child with IMAGe syndrome. Anesth Prog 2014; 61: 165-8.

9. Walker RW. Pulmonary aspiration in pediatric anesthetic practice in the UK: a prospective survey of specialist pediatric centers over a one-year period. Paediatr Anaesth 2013; 23: 702-11.

10. Monaca E, Fock N, Doehn M, Wappler F. The effectiveness of preformed tooth protectors during endotracheal 
intubation: an upper jaw model. Anesth Analg 2007; 105: 1326-32, table of contents.

11. Rabiner JE, Auerbach M, Avner JR, Daswani D, Khine H. Comparison of GlideScope Videolaryngoscopy to Direct Laryngoscopy for Intubation of a Pediatric Simulator by Novice Physicians. Emerg Med Int 2013; 2013: 407547.

12. Kim HJ, Kim JT, Kim HS, Kim CS. A comparison of GlideScope videolaryngoscopy with direct laryngoscopy for nasotracheal intubation in children. Paediatr Anaesth 2011;
21: $1165-6$

13. Moon YJ, Kim J, Seo DW, Kim JW, Jung HW, Suk EH, et al. Endotracheal intubation by inexperienced trainees using the Clarus Video System: learning curve and orodental trauma perspectives. J Dent Anesth Pain Med 2015; 15: 207.

14. Lee KH, You TM, Park W, Lee SH, Jung BY, Pang NS, et al. Protective dental splint for oroendotracheal intubation: experience of 202 cases. J Dent Anesth Pain Med 2015; 15: 17. 